\title{
Transfer of metformin into human milk
}

\author{
T. W. Hale ${ }^{1}$, J. H. Kristensen ${ }^{2}$, L. P. Hackett ${ }^{3}$, R. Kohan ${ }^{4}$, K. F. Ilett ${ }^{3,5}$ \\ ${ }^{1}$ Department of Pediatrics, Division of Clinical Pharmacology, Texas Tech University School of Medicine, Amarillo, Texas, USA \\ 2 Department of Pharmacy, King Edward Memorial and Princess Margaret Hospitals, Subiaco, Western Australia \\ ${ }^{3}$ Clinical Pharmacology and Toxicology Laboratory, The Western Australian Centre for Pathology and Medical Research, \\ Nedlands, Western Australia \\ ${ }^{4}$ Department of Neonatal Services, King Edward Memorial Hospital, Subiaco, Western Australia \\ ${ }^{5}$ Department of Pharmacology, University of Western Australia, Crawley, Western Australia
}

\section{Abstract}

Aims/hypothesis. The aim of this study was to characterize the milk-to-plasma ratio and infant dose for metformin in breastfeeding women, and to measure plasma concentrations and assess any effects in their infants. We hypothesized that metformin used by mothers is safe for their breastfed infants.

Methods. Seven women taking metformin (median dose $1500 \mathrm{mg}$ orally daily) and their infants were studied. Metformin concentrations in plasma and milk were measured by high performance liquid chromatography. Infant exposure was estimated as the product of estimated milk production rate and the average concentration of the drug in milk and also expressed as a percentage of the weight-normalized maternal dose.

Results. The mean milk-to-plasma ratio for metformin was 0.35 (95\%CI 0.2-0.5). The mean of its average concentrations in milk over the dose interval was
$0.27 \mathrm{mg} / \mathrm{l}(0.15-0.39 \mathrm{mg} / \mathrm{l})$. The absolute infant dose averaged $0.04 \mathrm{mg} \cdot \mathrm{kg}^{-1} \cdot \mathrm{day}^{-1}\left(0.02-0.06 \mathrm{mg} \cdot \mathrm{kg}^{-1} \cdot \mathrm{day}^{-1}\right)$ and the mean relative infant dose was $0.28 \%$ $(0.16-0.4 \%)$. Metformin was present in very low or undetectable concentrations in the plasma of four of the infants who were studied. No health problems were found in the six infants who were evaluated.

Conclusions/interpretation. The concentrations of metformin in breast milk were generally low and the mean infant exposure to the drug was only $0.28 \%$ of the weight-normalized maternal dose. As this is well below the $10 \%$ level of concern for breastfeeding, and because the infants were healthy, we conclude that metformin use by breastfeeding mothers is safe. Nevertheless, each decision to breastfeed should be made after conducting a risk:benefit analysis for each mother and her infant. [Diabetologia (2002) 45:1509-1514]

Keywords Metformin, human milk, milk-to-plasma ratio, infant dose, PCOS.
Diabetes mellitus affects more than $6 \%$ of the population of the United States, with the great majority having Type II (non-insulin-dependent) diabetes mellitus

Received: 3 May 2002 / Revised: 12 July 2002

Published online: 25 September 2002

(C) Springer-Verlag 2002

Corresponding author: Dr. T. W. Hale, Department of Pediatrics, Division of Clinical Pharmacology, Texas Tech University School of Medicine, Amarillo, Texas, USA.

E-mail: tom@ama.ttuhsc.edu

Abbreviations: AUC, Area under the curve from time 0 to time t; $\mathrm{C}_{\mathrm{av}}$, average concentration over a dose interval(s); LOD, limit of detection; PCOS, polycystic ovarian syndrome; hplc, high performance liquid chromatography; PGP, P-glycoprotein.
[1] In the past decade Type II diabetes has increased by $30 \%$ particularly in younger people, including pregnant women $[2,3]$. In addition, the use of metformin for treating polycystic ovarian syndrome (PCOS) has increased [4]. PCOS affects approximately 5\% of reproductive age patients. Metformin has been found to improve ovulatory function in insulin-resistant women with PCOS, to reduce first trimester miscarriage [5] and to decrease the progression from impaired glucose tolerance to Type II diabetes mellitus [6].

Therefore using metformin while breastfeeding is important, although to date no data are available on its transfer into human milk or to the breastfed infant.

Metformin is an antihyperglycaemic agent which improves glucose tolerance in patients with Type II di- 


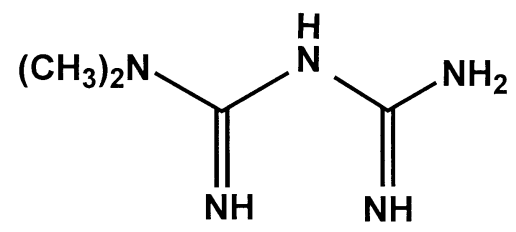

Fig. 1. Chemical structure of metformin (129 Da)

abetes; it lowers basal-increased and postprandial-increased blood glucose concentrations in patients with Type II diabetes. Its pharmacological mechanisms are different from other classes of oral antihyperglycaemic agents and are somewhat obscure. Rather than stimulating insulin release, metformin reduces hepatic glucose production $[7,8]$ which can then facilitate secondary disposal of glucose in peripheral tissues [9]. Ultimately metformin increases insulin-stimulated glucose uptake in skeletal muscle and adipocytes [7, 10, 11]. Unlike sulfonylureas, metformin does not produce hypoglycaemia in diabetic or healthy patients and circulating insulin concentrations tend to decline [6].

Metformin (Fig. 1) is a small amphoteric molecule (129 Da) with pKa values of 2.8 and $11.5[12,13]$. These physicochemical properties give metformin high water solubility and very low lipid solubility. It is incompletely absorbed from the GI tract (bioavailability $50-60 \%$ [14]), with the majority absorbed within $6 \mathrm{~h}$ after ingestion [14]. Sustained release formulas also have a low oral availability suggesting that absorption occurs mostly in the small intestine [15]. It has a distribution volume of about $41 / \mathrm{kg}[14,16]$, is not bound to plasma proteins, and after oral administration it is eliminated in urine and faeces [14] with an elimination half-life of about 4 to $5 \mathrm{~h}[14,16,17]$.

We describe the transfer of metformin into human milk in seven breastfeeding women. The concentration of drug in milk was measured either in a single milk sample (one patient), or from multiple samples taken over one or more dose intervals (six women), and was later used to calculate the infant dose.

\section{Subjects and methods}

Study protocol. Seven breastfeeding women and their infants were enrolled in the study. The study design was approved by the Research and Ethics Committee of King Edward Memorial and Princess Margaret Hospitals and also by the Texas Tech University Institutional Review Board. Written informed consent was obtained from the women.

Data collection. In two patients, a detailed study of milk and plasma metformin concentrations (measurement of area under the concentration-time curve, AUC) was made over an 8-h dose interval at steady-state. Briefly, venous blood samples $(8 \mathrm{ml}$; heparinised) were collected from the women via a forearm cannula at $0,1,2,3,4,5,6$ and $8 \mathrm{~h}$ post-dose. At the same time intervals, a representative milk sample $(20 \mathrm{ml}$ from both breasts; usually, an approximately equal mix of milk collected before and after feeding) was collected using an electric or manual breast pump. Both women gave consent for a venous blood sample (0.5-1 ml, heparinised) to be taken by venipuncture from their infants. In four patients, metformin concentration in milk (AUC measurement) was measured using samples (20 ml each; an approximately equal mix of milk collected before and after feeding) collected over three sequential 8-h dose intervals at steady-state. Sampling times for breast milk were just before the first of the three doses (500 $\mathrm{mg}$ thrice daily) and at 2, 4, 6, 8 and $24 \mathrm{~h}$ after the first of the three doses. A single blood sample was taken from each of these women $2 \mathrm{~h}$ after the first of the three doses, and two women gave consent for a venous blood sample $(0.5-1 \mathrm{ml}$, heparinised) to be taken by venipuncture from their infants. In one patient who was also at steady-state, single blood and milk samples were obtained $2 \mathrm{~h}$ after the morning dose of metformin. For all studies, the health and wellbeing of the infants were evaluated by interviewing the mothers and when possible by a full clinical examination by a specialist neonatologist or paediatrician. Infant body weight for age was checked against sex-specific population percentile graphs [18] and in the two full AUC studies, a Denver Developmental Screening Test was done on both infants $[19,20]$.

Materials. Metformin hydrochloride (catalogue no. D-5035) and dihydrocodeine bitartrate (catalogue no. 017772) standards were obtained from Sigma Chemical Company, Castle Hill, Australia and Alltech Associates Australia, Box Hill, Australia. All solvents and other chemicals were of analytical or high performance liquid chromatography (hplc) grade.

High performance liquid chromatography. Aliquots of plasma $(0.1 \mathrm{ml})$ were added to $1 \mathrm{ml}$ acetonitrile (containing $2 \mu \mathrm{g}$ of dihydrocodeine as internal standard) whilst vortexing vigorously for $30 \mathrm{~s}$ to precipitate proteins. After centrifugation at $1500 \mathrm{~g}$ for $5 \mathrm{~min}$, the supernatant was transferred to a clean tube and extracted with $1 \mathrm{ml}$ hexane by vortexing for $10 \mathrm{~s}$. After a further centrifugation as above, the hexane layer was discarded and the acetonitrile:water layer was evaporated to dryness at $45^{\circ} \mathrm{C}$ under a stream of dry nitrogen. Extracts were reconstituted in $0.2 \mathrm{ml}$ of the hplc mobile phase and $0.05 \mathrm{ml}$ aliquots were injected onto the hplc column. The metformin concentration in patient plasma samples was interpolated from a standard curve for metformin in plasma (Y-axis, peak height ratio metformin:dihydrocodeine; X-axis, added concentration of metformin) that were prepared with each batch of samples. Standard curves were linear over a range from $0.1-5 \mathrm{mg} / \mathrm{l}$ with correlation coefficients of 0.998 or better.

Concentrations of metformin in milk were measured by hplc, using the method of addition as follows. Briefly, metformin concentrations were determined by taking four equal aliquots of each milk sample, and spiking three of these with increasing concentrations of authentic metformin. A fraction of each of these milk samples $(0.1 \mathrm{ml})$ was then added to $5 \mathrm{ml}$ of acetonitrile (containing $1 \mu \mathrm{g}$ dihydrocodeine as internal standard) whilst vigorously vortexing for $30 \mathrm{~s}$. After centrifugation as above, the acetonitrile phase was evaporated to dryness as above and extracts were reconstituted in $0.2 \mathrm{ml}$ distilled water and $1 \mathrm{ml}$ of water-saturated isopropyl ether. After vortexing for $10 \mathrm{~s}$, and centrifuging as above for $15 \mathrm{~min}$, the ether phase was aspirated to waste, and $0.07 \mathrm{ml}$ aliquots of the aqueous phase were injected onto the hplc column. Standard curves (Y-axis, peak height ratio metformin:dihydrocodeine; $\mathrm{X}$-axis, added metformin concentration), were constructed for each individual sample and the concentration of metformin originally present in each sample was measured from the neg- 
ative $\mathrm{X}$-axis intercept. The curves usually ranged from $0.03-1.2 \mathrm{mg} / \mathrm{l}$ with a typical correlation coefficient of more than 0.996.

Analytes were resolved on a LiChrospher RP_Select B column ( $5 \mu \mathrm{m}$; $4.6 \mathrm{~mm} \times 250 \mathrm{~mm}$; E. Merck, Damsdart, FDR) using a mobile phase of $72 \%$ acetonitrile in $0.01 \mathrm{~mol} / \mathrm{l}$ of potassium dihydrogen phosphate buffer (previously adjusted to $\mathrm{pH} 8$ with diethylamine) at a flow of $1.5 \mathrm{ml} / \mathrm{min}$. Eluting peaks were detected and quantified by their UV absorbance at $235 \mathrm{~nm}$ using a Waters Associates Model 2487 dual wavelength absorbance detector. The precision of the assay was evaluated as the coefficient of variation (CV) for both plasma and milk. For milk ( $n=3-4)$, at $0.05 \mathrm{mg} / \mathrm{l}, 0.2 \mathrm{mg} / \mathrm{l}$ and $1 \mathrm{mg} / \mathrm{l}$, intra-day CVs were $11.7 \%, 6.1 \%$ and $5.1 \%$ respectively and inter-day CVs were $12.1 \%, 7.2 \%$ and $4.1 \%$ respectively. The limit of detection (LOD) was $0.01 \mathrm{mg} / \mathrm{l}$ for plasma and milk.

Data analysis. Data have been summarised as mean (95\% CI, SEM, or range), or median (range) as appropriate.

Calculation of milk/plasma ratio and infant dose. AUCs were calculated using the pharmacokinetic program Topfit (log trapezoidal rule) [21]. Milk-to-plasma ratios were calculated using AUC data (two patients), or from the ratio of the average concentration in milk $\left[\mathrm{C}_{\mathrm{av}}=\mathrm{AUC}_{0-\tau} / \tau\right.$, where $\tau=$ duration of the dose interval(s) in h] to the "corrected" 2 -h plasma concentration (four patients). Since the measured 2-h plasma concentrations would over-estimate the true $\mathrm{C}_{\mathrm{av}}$, they were corrected by dividing by a factor equal to the mean of the 2-h plasma concentration divided by the average plasma concentration for the two patients who had full AUC data over an 8-h dose interval (factor $=1.27$ ). In one patient, the milk-to-plasma ratio was calculated from a single set of milk and plasma concentrations measured at $2 \mathrm{~h}$, with correction of the plasma concentration as above. An infant milk intake of $0.151 \cdot \mathrm{kg}^{-1}$. day ${ }^{-1}$ was assumed [22] and this value was multiplied by the milk $\mathrm{C}_{\mathrm{av}}$ to give the absolute infant dose of metformin in $\mathrm{mg} \cdot \mathrm{kg}^{-1} \cdot \mathrm{day}^{-1}$. The latter value was then expressed as a percentage of the weight-normalised maternal dose $\left(\mathrm{mg} \cdot \mathrm{kg}^{-1} \cdot \mathrm{day}^{-1}\right)$ to yield the measure known as relative infant dose.

\section{Results}

The breastfeeding women had a mean age of 34 years (range 26-38 years) and a mean body weight of $97 \mathrm{~kg}$ (range $73-116 \mathrm{~kg}$ ). Their infants, including one pair of male twins (patient no. 2) were four male infants and four female infants with a mean age of 14.3 months (range 5-25 months) and a mean body weight of $10.8 \mathrm{~kg}$ (range $6.5-15 \mathrm{~kg}$ ). Six women took $500 \mathrm{mg}$ of metformin orally, thrice daily before meals, while one (no. 6) took $500 \mathrm{mg}$ of a slow release metformin formula once daily. All had been on these dose regimens for at least 3 weeks at the time of the study and their plasma and milk metformin concentrations can reasonably be expected to have been at steady-state. When normalized to body weight, the median daily metformin dose was $14 \mathrm{mg} \cdot \mathrm{kg}^{-1} \cdot \mathrm{day}^{-1}$ (range $6.9-20 \mathrm{mg} \cdot \mathrm{kg}^{-1} \cdot \mathrm{day}^{-1}$ ). Five of the women were taking metformin for treatment of PCOS and two for treatment of Type II diabetes. All had begun therapy after the birth of their infants.
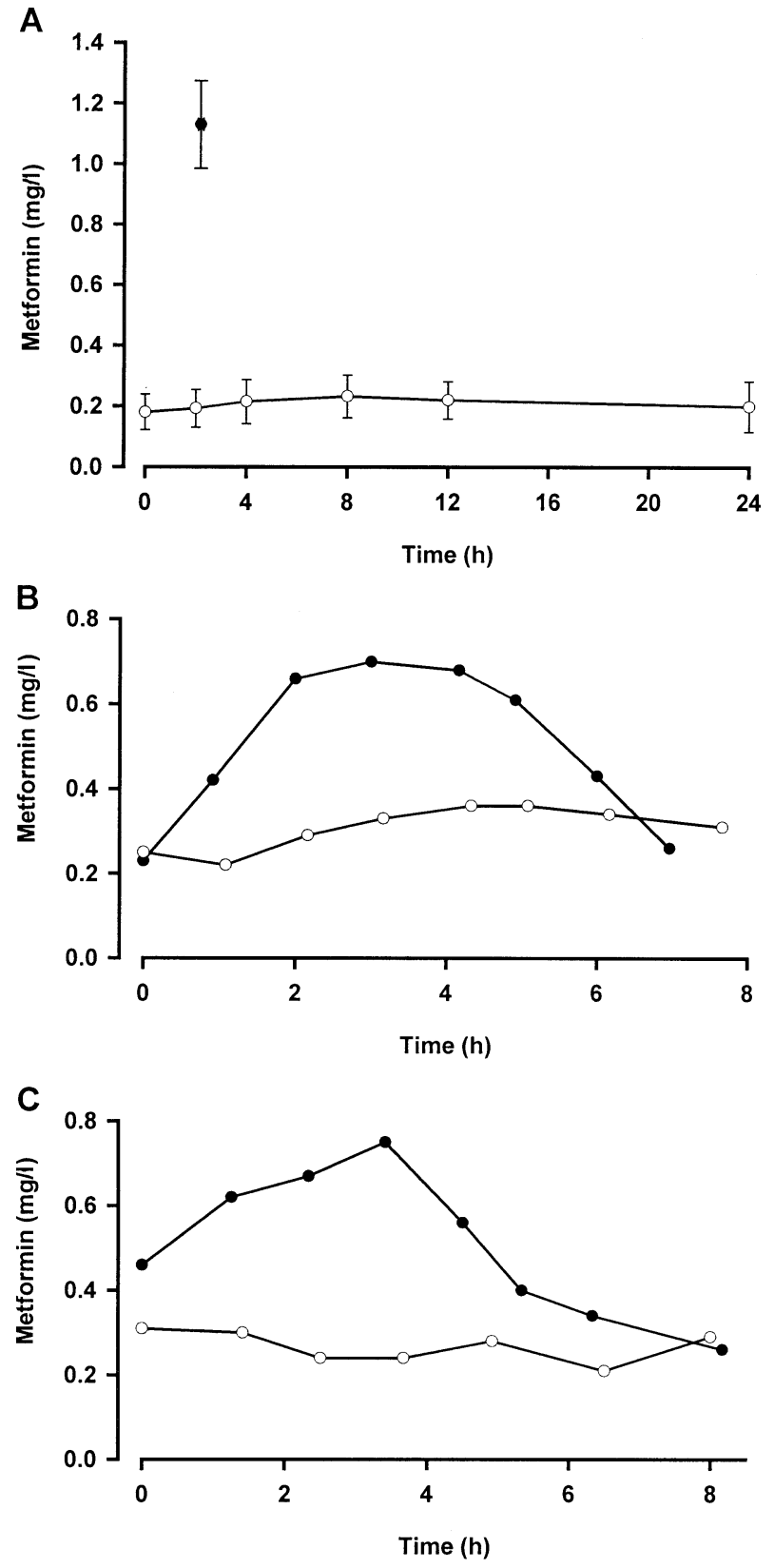

Fig. 2A-C. Concentration-time profiles for metformin in plasma (O) and milk $(\bigcirc)$ during administration of $500 \mathrm{mg}$ metformin orally thrice daily. A Data for 5 patients as mean \pm SEM, with sampling over $24 \mathrm{~h}$ covering three sequential dose intervals. B, C Individual data for patients 1 and 2 respectively, with sampling over an 8-h dose interval

Plasma and milk concentration-time profiles for metformin in six of the seven patients are shown in Fig. 2A-C. These plots show that the concentration of metformin in milk has a flat profile. In contrast, the plasma concentration-time profile measured over a single dose interval in two patients showed a clear peak at about 3 to $4 \mathrm{~h}$ after dose. The milk and plasma concentration data and milk-to-plasma ratio for all patients are summarized in Table 1. Plasma concentrations were within the expected range for patients taking a dose of $1500 \mathrm{mg}$ per day of the drug [17]. Milk 
Table 1. Metformin concentrations in milk and maternal plasma, milk-to-plasma (M/P) ratio, calculated infant doses and infant plasma concentrations

\begin{tabular}{|c|c|c|c|c|c|c|}
\hline \multirow{2}{*}{$\begin{array}{l}\text { Mother and } \\
\text { infant no. }\end{array}$} & \multicolumn{2}{|c|}{ Metformin (mg/l) } & \multirow{2}{*}{$\begin{array}{l}\mathrm{M} / \mathrm{P} \\
\text { ratio }\end{array}$} & \multicolumn{2}{|l|}{ Infant dose } & \multirow{2}{*}{$\begin{array}{l}\text { Infant plasma } \\
\text { concentration } \\
(\mathrm{mg} / \mathrm{l})\end{array}$} \\
\hline & Milk & Plasma & & $\begin{array}{l}\text { Absolute } \\
\left(\mathrm{mg} \cdot \mathrm{kg}^{-1} \cdot \mathrm{day}^{-1}\right)\end{array}$ & $\begin{array}{l}\text { Relative to } \\
\text { weight-normalized } \\
\text { maternal dose } \\
(\%)\end{array}$ & \\
\hline 1 & $0.30^{\mathrm{a}}$ & $0.52^{\mathrm{a}}$ & 0.58 & 0.045 & 0.23 & 0.08 \\
\hline 4 & $0.24^{b}$ & $0.76^{d}$ & 0.32 & 0.036 & 0.24 & - \\
\hline 5 & $0.15^{b}$ & $0.96^{d}$ & 0.16 & 0.023 & 0.15 & - \\
\hline 6 & $0.07^{b}$ & $0.52^{\mathrm{d}}$ & 0.13 & 0.011 & 0.50 & $<0.01^{\mathrm{e}}$ \\
\hline 7 & $0.43^{c}$ & $1.15^{\mathrm{d}}$ & 0.37 & 0.064 & 0.15 & $<0.01 \mathrm{e}$ \\
\hline $\begin{array}{l}\text { Mean } \\
(95 \% \text { CI })\end{array}$ & $\begin{array}{l}0.27 \\
(0.15-0.39)\end{array}$ & $\begin{array}{l}0.79 \\
(0.26-1.05)\end{array}$ & $\begin{array}{l}0.35 \\
(0.2-0.5)\end{array}$ & $\begin{array}{l}0.04 \\
(0.02-0.06)\end{array}$ & $\begin{array}{l}0.28 \\
(0.16-0.4)\end{array}$ & \\
\hline
\end{tabular}

a Average concentrations estimated from $\mathrm{AUC}_{0,8}$

$\mathrm{b}$ Average concentrations estimated from $\mathrm{AUC}_{0,24}$

c Single concentration measurement at $2 \mathrm{~h}$ after a dose

d Single measured concentration at $2 \mathrm{~h}$ after a dose. Corrected to an estimated average plasma concentration by dividing the

metformin concentrations were a mean of threefold lower than those in plasma, as reflected in the mean milk-to-plasma ratio of 0.35 . Calculation of the theoretical milk-to-plasma ratio for metformin was undertaken by a method described previously [23] using a $\mathrm{pKa}$ of 11.5 [12], a $\log _{10}(\mathrm{P})_{\mathrm{pH}} 7$ value of -1.43 [13], a plasma protein binding of $0 \%$ [14] and a milk $\mathrm{pH}$ of 7.2, yielding a milk-to-plasma ratio of 2.93 .

Calculated infant dose data are also summarized in Table 1. The mean absolute infant dose was $0.04 \mathrm{mg} \cdot \mathrm{kg}^{-1} \cdot \mathrm{day}^{-1}$ and when compared to the weight-normalized maternal dose yielded a mean relative infant dose of $0.28 \%$. This very low dose exposure was confirmed by undetectable concentrations of metformin in plasma from two of the four infants from whom we were permitted to take a blood sample (both at $2.5 \mathrm{~h}$ after the maternal dose), and low plasma concentrations of $0.08 \mathrm{mg} / \mathrm{l}$ and $0.05 \mathrm{mg} / \mathrm{l}$ at 5.3 and $6 \mathrm{~h}$ respectively, after the maternal dose for the other two infants. Moreover, in the opinion of the mothers $(n=4)$ and/or physicians $(n=2)$, the infants (data unavailable for infant of patient no. 3) were healthy and progressing as expected. In two infants (of patients no. 1 and no. 2), a Denver Developmental Screening test was done which showed that both were normal for age in all respects.

\section{Discussion}

The decision to use antidiabetic medications in breastfeeding mothers is quite complex. The importance of breastfeeding to the individual mother, the enormous benefits of nursing the infant, and the adverse effects measured concentration by a factor equal to the mean of the 2-h plasma concentration divided by the average plasma concentration for patients 1 and 2 who had full AUC data over an 8-h dose interval $($ factor $=1.27$ )

e Assay LOD

of the individual medications must be carefully considered in the risk/benefit analysis of prescribing antidiabetic agents to breastfeeding mothers. In addition, the mechanism of action of the drug can be important. Although some antidiabetic medications (e.g. sulfonylureas) reduce plasma glucose concentrations in treated patients, the biguanides (e.g. metformin) do not alter plasma glucose concentrations in healthy people. Thus, biguanides can be preferred in breastfeeding mothers.

Presently, the use of metformin to treat PCOS is increasing as recent evidence shows metformin stabilizes endocrine function in pregnant and non-pregnant women [24]. Because this major endocrinopathy occurs in approximately 5\% of the reproductive-age population and is a major cause of infertility [4], its use in breastfeeding mothers is rapidly increasing. Thus, five of the seven patients in this study were being treated for PCOS.

Drugs transfer into human milk largely as a function of lipophilicity, molecular weight, protein binding, maternal drug concentration in plasma, and drug $\mathrm{pKa}$ [25]. Predicting the milk-to-plasma ratio on theoretical grounds has been recommended as a means of estimating infant exposure when maternal plasma concentrations of the drug are the only data available [26]. Using this method we calculated a milk-to-plasma ratio of 2.93 for metformin. This value is 8.3 -fold higher than the measured mean milk-to-plasma ratio of 0.35 in our study. Thus, our data suggest that metformin-transfer into milk does not behave as predicted since we found that milk concentrations were relatively flat throughout a dose interval, while maternal plasma concentrations showed a peak at about 3 to $4 \mathrm{~h}$ af- 
ter dose. In our experience, the algorithm for calculating milk-to-plasma ratio for basic drugs is usually quite robust. Others [27] also observed a similar low milk-to-plasma ratio (0.6) and relative infant dose $(0.2 \%)$ for metformin and suggested [28] that the low dose might arise if metformin were a substrate for a $\mathrm{P}$ glycoprotein type pump (PGP) located in the breast lobular epithelial cells. However we are unaware of any evidence that metformin is a PGP substrate, or that PGPs exist in breast tissues. We suggest that the discrepancy in the calculated milk-to-plasma ratio is more likely to be caused by metformin's very high pKa (relevant value $=11.5$ ) and very low lipid solubility, which are at the extremes of the range of values used when the original predicted algorithm was derived [26]. These physicochemical properties would be expected to make it very difficult for the drug to passively diffuse across lipoprotein membranes which is supported by its low (50-60\%) oral bioavailability [14] and the observation that its oral bioavailability is not affected by co-administration with a high-fat meal [29] The fact that the milk and plasma concentrationtime profiles were very different, highlights the importance of using AUC data to estimate both the milkto-plasma ratio and infant dose. In terms of the absolute value of the milk-to-plasma ratio, we were only able to calculate AUC-based estimates in two patients, while a single point estimate or a corrected single point estimate was calculated in the other five patients. $\mathrm{M} / \mathrm{P}_{\mathrm{AUC}}$ values $(0.5$ and 0.58$)$ were somewhat higher than those from the single point estimates (range 0.13-0.37), and are more robust in our view. Nevertheless, the milk-to-plasma ratio is primarily of interest in characterising the mechanism of metformin transfer into milk and was not required for the estimation of infant dose that was calculated from AUC milk in all seven patients.

Safety of drug use during breastfeeding is assessable either by comparing the absolute infant dose to usual therapeutic doses in infants, or by comparing the infant dose to the mother's dose (relative infant dose) [25]. The mean absolute and relative infant doses calculated for metformin were $0.04 \mathrm{mg} \cdot \mathrm{kg}^{-1} \cdot \mathrm{day}^{-1}$, and $0.28 \%$ (of the maternal weight-normalised dose) respectively. There is no comparison for absolute infant dose since metformin is not used in infants. However, the relative infant dose is well below the recommended $10 \%$ "level of concern" [22], and the safety of maternal metformin use for the breastfed infant therefore appears to be high. Moreover, metformin has a low oral availability in adults [14] and if availability is similarly low in infants the dose of metformin actually delivered via the milk ingested by the infant would be further decreased. In two infants where metformin was detected, the plasma concentrations were approximately $15 \%$ and $10 \%$ of those in maternal plasma, while in two other infants the drug was not detected. These observations were consistent with our expecta- tion of very low concentrations of exposure via milk. The absence of any adverse effect in the infants is also reassuring, although our data on this area are sparse and somewhat subjective. Therefore, we recommend that women who need to take metformin for control of Type II diabetes or PCOS should be encouraged to breastfeed their infants. As is appropriate with breastfeeding when maternal drug therapy is necessary, a thorough risk-benefit analysis of proposed therapy should be made before breastfeeding begins, and frequent regular checks of the infant's progress should subsequently be made. Since metformin is largely excreted via the kidneys, particular caution should be exercised where the infant renal function is low or compromised (e.g. in premature neonates, or in rare cases of renal failure).

Acknowledgements. We acknowledge funding from the Women and Infants Research Foundation, Western Australia. We thank R. Barrett-Lennard, N. Butcher and M. Paech for assistance with data collection, and Medela, USA for the loan of a Baby Weigh Scale.

\section{References}

1. Harris MI (1998) Diabetes in America: epidemiology and scope of the problem. Diabetes Care 21 [Suppl 3]: C11-C14

2. Rosenbloom AL, House DV, Winter WE (1998) Non-insulin dependent diabetes mellitus (NIDDM) in minority youth: research priorities and needs. Clin Pediatr (Phila) 37: $143-152$

3. Rosenbloom AL, Joe JR, Young RS, Winter WE (1999) Emerging epidemic of type 2 diabetes in youth. Diabetes Care 22: 345-354

4. Knochenhauer ES, Key TJ, Kahsar-Miller M, Waggoner W, Boots LR, Azziz R (1998) Prevalence of the polycystic ovary syndrome in unselected black and white women of the southeastern United States: a prospective study. J Clin Endocrinol Metab 83: 3078-3082

5. Glueck CJ, Phillips H, Cameron D, Sieve-Smith L, Wang P (2001) Continuing metformin throughout pregnancy in women with polycystic ovary syndrome appears to safely reduce first-trimester spontaneous abortion: a pilot study. Fertil Steril 75: 46-52

6. Inzucchi SE (2002) Oral antihyperglycemic therapy for type 2 diabetes: scientific review. JAMA 287: 360-372

7. Hundal RS, Krssak M, Dufour S et al. (2000) Mechanism by which metformin reduces glucose production in type 2 diabetes. Diabetes 49: 2063-2069

8. Inzucchi SE, Maggs DG, Spollett GR et al. (1998) Efficacy and metabolic effects of metformin and troglitazone in type II diabetes mellitus. N Engl J Med 338: 867-872

9. Bailey CJ, Turner RC (1996) Metformin. N Engl J Med 334: 574-579

10. Wiernsperger NF, Bailey CJ (1999) The antihyperglycaemic effect of metformin: therapeutic and cellular mechanisms. Drugs 58 [Suppl 1]: 31-39, discussion 75-82, 31-39

11. Perriello G, Misericordia P, Volpi E et al. (1994) Acute antihyperglycemic mechanisms of metformin in NIDDM. Evidence for suppression of lipid oxidation and hepatic glucose production. Diabetes 43: 920-928 
12. Baselt RC (2000) Metformin. In: Disposition of toxic drugs and chemicals in man, 5th edn. Chemical Toxicology Institute, Foster City, pp 523-524

13. Jack DB (1992) Handbook of clinical pharmacokinetic data. Macmillan, Basingstoke

14. Tucker GT, Casey C, Phillips PJ, Connor H, Ward JD, Woods HF (1981) Metformin kinetics in healthy subjects and in patients with diabetes mellitus. Br J Clin Pharmacol 12: $235-246$

15. Gusler G, Gorsline J, Levy G et al. (2001) Pharmacokinetics of metformin gastric-retentive tablets in healthy volunteers. J Clin Pharmacol 41: 655-661

16. Yuen KH, Wong JW, Billa N, Julianto T, Toh WT (1999) Bioequivalence of a generic metformin tablet preparation. Int J Clin Pharmacol Ther 37: 319-322

17. Hirschberg Y, Karara AH, Pietri AO, McLeod JF (2000) Improved control of mealtime glucose excursions with coadministration of nateglinide and metformin. Diabetes Care 23: 349-353

18. Hamill PV, Drizd TA, Johnson CL, Reed RB, Roche AF (1977) NCHS growth curves for children birth-18 years. United States. Vital \& Health Statistics - Series 11: data from the national health survey-74, pp 1-74. National Center for Health Statistics, Washington DC, USA

19. Frankenburg WK, Dodds JB (1967) The Denver development screening test. J Pediatr 71: 181-191

20. Rossiter EJ (1993) The use of developmental screening and assessment instruments by paediatricians in Australia. J Paediatr Child Health 29: 357-359

21. Thomann P (1993) Non-compartmental analysis methods manual. In: Heinzel G, Woloszcak R, Thomann P (eds) TopFit 2.0 Pharmacokinetic and pharmacodynamic data analysis system for the PC. 1st edn. Gustav Fischer, Stuttgart, pp 5-66

22. Bennett PN (1996) Use of the monographs on drugs. In: Bennett PN (ed) Drugs and human lactation, 2nd edn. Elsevier, Amsterdam, pp 67-74

23. Begg EJ, Atkinson HC, Duffull SB (1992) Prospective evaluation of a model for the prediction of milk:plasma drug concentrations from physicochemical characteristics. Br J Clin Pharmacol 33: 501-505

24. Kocak M, Caliskan E, Simsir C, Haberal A (2002) Metformin therapy improves ovulatory rates, cervical scores, and pregnancy rates in clomiphene citrate-resistant women with polycystic ovary syndrome. Fertil Steril 77: 101-106

25. Hale TW, Ilett KF (2001) Drug therapy and breastfeeding: from theory to clinical practice. The Parthenon Publishing Group, London

26. Atkinson HC, Begg EJ (1990) Prediction of drug distribution into human milk from physicochemical characteristics. Clin Pharmacokinet 18: 151-167

27. Zhang M, Moore GA, Lever M, Gardiner SJ, Kirkpatrick CM, Begg EJ (2002) Rapid and simple high-performance liquid chromatographic assay for the determination of metformin in human plasma and breast milk. J Chromatogr B Analyt Technol Biomed Life Sci 766: 175-179

28. Gardiner SJ (2001) Drugs in lactation. Proceedings of the Australasian Society of Clinical and Experimental Pharmacologists and Toxicologists 9: 9

29. Marathe PH, Arnold ME, Meeker J, Greene DS, Barbhaiya RH (2000) Pharmacokinetics and bioavailability of a metformin/glyburide tablet administered alone and with food. J Clin Pharmacol 40: 1494-1502 